\title{
¿Cómo abordar la dimensión socioafectiva en la enseñanza remota de emergencia?
}

\author{
J. Escamilla, R. García, C. Moreno, J. Osoria, J. Peña, A. González, J. Rodríguez, \\ L. Salazar, R. Saldivar, V. Luna, O. Briz, A. García, N. Galván, M. Garrido, H. \\ Infanzón, C. Rivero, K. Yap y C. Zavala
}

\begin{abstract}
Resumen
Para continuar la docencia durante la etapa de confinamiento generada por la pandemia, los profesores tuvimos que seguir enseñando en una modalidad que ha sido llamada enseñanza remota de emergencia. Enfrentamos el reto de repensar los métodos de enseñanza y aprendizaje, así como aspectos clave de la dimensión socioafectiva que son de gran relevancia para el éxito en esta transición.

Con la intención de contribuir a la preparación de los profesores en esta transición, algunos integrantes de las instituciones que conforman la Red de Innovación Educativa 360 (RIE360) hemos preparado una serie de recomendaciones para atender la dimensión socioafectiva en la enseñanza remota de emergencia. Presentamos una descripción de las etapas por las que podría transitar el estudiante ante el cambio de modalidad; se exponen algunas ideas para involucrar y motivar a los estudiantes en su aprendizaje, así como pautas para establecer una relación afectiva en entornos virtuales.
\end{abstract}

Palabras clave: enseñanza remota de emergencia, enseñanza virtual, enseñanza en línea, contingencia educativa, dimensión socioafectiva.

\section{HOW TO ADDRESS THE SOCIO-AFFECTIVE DIMENSION IN EMERGENCY REMOTE TEACHING?}

\begin{abstract}
To continue teaching during the confinement phase of the pandemic, higher education teachers have had to keep up teaching at a distance, in a model that has been identified as remote emergency education. We have faced the challenge of revising our teaching and learning strategies, as well as the socio-emotional aspects that impact students success in this transition. Members of the Network for Educational Innovation (Red de Innovación Educativa 360, o RiE360) have prepared a series of recommendations to address the social dimension in teaching and learning during the pandemic. We describe the stages that students go through as they move on to this new educational model; then we present ideas to engage and motivate students in their learning process, as well as to establish an effective relationship with them.
\end{abstract}

Keywords: emergency remote teaching, educational contingency, online teaching, teacher-student relationship, socio-affective dimension. 
José G. Escamilla (ITESM); Rubén García y Carlos Iván Moreno (UdeG); Jhonatan O. Osoria, Armando Peña, Arely González, Jessica M. Rodríguez, Lorena E. Salazar, Roxana Saldivar (UANL); Verónica Luna, Oliva Briz, Angélica García, Nancy A. Galván, Mariana Garrido, Héctor M. Infanzón, Carlos A. Rivero, Kweilan Yap y Carmen Zavala (UnAm).

La Red de Innovación Educativa (RIE360), es un grupo interinstitucional de trabajo conformado por instituciones mexicanas de educación superior, públicas y privadas. Para mayor información sobre los antecedentes de esta red, se sugiere consultar el artículo publicado en el volumen 19, número 1, enero-febrero 2018 de la RDu: http://doi.org/10.22201/codeic.16076079e.2018.v19n1.a5

\section{Datos de contacto}

Verónica Luna de la Luz: veroluluz@gmail.com

\section{Introducción}

Con el fin de dar continuidad a las clases ante la contingencia sanitaria, la comunidad educativa ha adaptado diferentes estrategias hacia la enseñanza remota de emergencia. Al transitar a espacios de aprendizaje que implican interacciones en línea con los estudiantes, es necesario que, como profesores, además de la preparación didáctica también consideremos los aspectos socioafectivos que invariablemente están presentes y que influyen en gran medida en el interés, motivación y autorregulación del estudiante (Wosnitza 7 volet, 2005).

A la luz de lo anterior, integrantes de la Red de Innovación Educativa (RIE360) hemos preparado una serie de recomendaciones para que los profesores consideren la dimensión sociafectiva en la enseñanza y el aprendizaje en esta transición. Primero, presentamos una descripción de las etapas por las que podrían transitar los estudiantes ante el cambio de modalidad; posteriormente, ideas para involucrar y motivarlos en su aprendizaje, así como para establecer una relación efectiva con ellos en los entornos virtuales. 


\section{Etapas de un estudiante ante el cambio de paradigma}

La pandemia ha trastocado nuestra vida a nivel personal y profesional, nos ha orillado a repensar nuestras acciones como profesores, poniéndonos en una encrucijada, en donde prevalece la incertidumbre sobre el futuro. Encontramos que estar preparados es la mejor estrategia para afrontar los retos actuales y venideros. Esto conlleva pensar más profundamente cómo estamos viviendo este cambio de paradigma hacia la educación en línea y cómo lo están afrontando los estudiantes.

Las situaciones que implican un cambio abrupto a nuestra rutina o modo de vivir han sido estudiadas desde diferentes disciplinas. A continuación, abordamos una perspectiva psicológica y, después, una del ámbito de la educación en línea. Desde la psicología se retoman las fases de la crisis, todavía vigentes, planteadas por Caplan (1961). Una crisis puede estar ocasionada por la situación personal de cada individuo, aunado al contexto de cambio de paradigma formativo. Se divide en:

a) Shock o impacto ante un evento súbito. Se experimenta irritación, impotencia, desamparo, ansiedad ante la situación.

b) Desorganización crítica. El individuo trata de hacer uso de estrategias habituales para sobrellevar el evento crítico, entra a un estado de indefensión en donde se percata de que no está preparado para hacerle frente a la situación, o quizá encuentre apoyo para darle un buen cause de afrontamiento.

c) Resolución. Momento en el que es posible que se active la creatividad para plantearse estrategias de afrontamiento, para aprender de y con otros.

d) Decisión final. A partir del éxito obtenido de la puesta en marcha de las acciones anteriores, el individuo toma una decisión sobre cómo continuar y salir paulatinamente del estado de crisis.

En lo referente al cambio de un modelo educativo tradicional a la educación en línea, es posible que el estudiante viva un proceso de adaptación, como lo señaló hace algunas décadas Woods (1994). Al inicio, posee un nivel de desempeño acorde con los conocimientos y habilidades desarrollados en un modelo educativo tradicional o típico; sin embargo, a medida que interactúa con el modelo de aprendizaje no presencial comienza a transitar por las siguientes etapas:

1. El estudiante experimenta una crisis debido al conflicto de paradigmas que ocurre cuando una persona ha vivido y aprendido bajo un modelo determinado durante un tiempo prolongado, y de pronto se encuentra inmerso en uno nuevo. Esto implica un cambio brusco que trastoca su estabilidad.

2. Inmediatamente después de esta crisis, el estudiante toma una postura negativa ante el nuevo paradigma educativo, que le obliga a salir de su zona segura. 
3. El estudiante inicia un descenso en su estado de ánimo de forma paulatina, constante y profunda, que se manifiesta en una pérdida de confianza y síntomas como: miedo, angustia, culpabilidad, enojo y resentimiento.

4. Como consecuencia, el estudiante, desarrolla una actitud de resistencia: se encuentra bloqueado. Manifiesta aislamiento y se plantea abandonar el trabajo que le implica el nuevo paradigma: contempla la posibilidad de renunciar.

5. El estudiante ha llegado al nivel más profundo de desánimo y al grado mínimo de desempeño. Toma entonces una actitud de rendición al considerar una mínima posibilidad de éxito.

6. Sin embargo, debido a la riqueza de recursos con los que puede contar la persona ante el fracaso, el estudiante inicia un ascenso sorprendente, buscando oportunidades en esa nueva realidad que ahora experimenta. No se da por vencido, poco a poco comienza la lucha por afirmarse en el nuevo paradigma educativo y por salir adelante a pesar de las dificultades que ha encontrado en el camino.

7. Como resultado de esta actitud de lucha y de visión positiva ante el cambio, el estudiante va logrando pequeños éxitos en su desempeño, su nivel de confianza se eleva y la aceptación del nuevo paradigma inicia.

8. Finalmente, el estudiante tiene éxito con el nuevo modelo y consigue un nivel de desempeño muy favorable, que incluso puede ser superior al que tenía en el modelo educativo anterior. El estudiante ha integrado sus experiencias y vivencias, comprobando que esta nueva forma de aprender da resultado: jesto funciona!

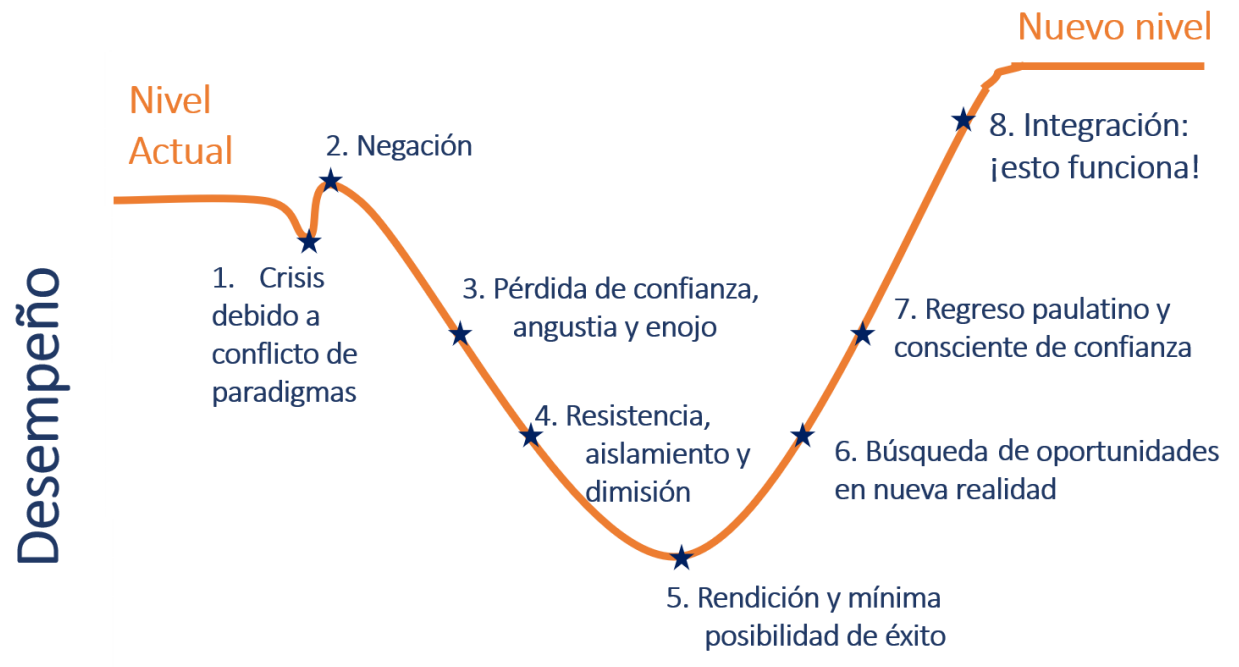

Figura 1. Etapas del estudiante ante el cambio de paradigma educativo (Woods, 1994, traducción propia).

\section{Tiempo (un semestre máx.)}

Es importante hacerle notar al estudiante la alta probabilidad de que transite por estas etapas; incluso, pedirle que identifique en cuál de ellas considera que 
se encuentra, y animarlo a continuar y a buscar estrategias para enfrentarlas y superarlas. Hay que ofrecerle apoyo para revisar cómo va su proceso de adaptación al nuevo paradigma, haciéndole saber que eventualmente podrá retomar un nivel de desempeño alto, una vez que internalice la enseñanza y el aprendizaje en línea.

\section{Apoyar a los estudiantes para que tengan éxito en la modalidad en línea}

Un curso en línea no es fácil. No lo es para los docentes ni para los estudiantes que hemos tenido la experiencia, mucho menos para quienes nunca la han vivido. No podemos pasar por alto que no será un solo curso el que los estudiantes llevarán, sino toda la carga académica del semestre o año en curso, lo que vuelve el proceso más retador. Es por eso que, a continuación, presentamos algunas recomendaciones para los estudiantes, con el fin de que el desarrollo del curso en línea tenga un final satisfactorio:

- Elegir un espacio donde se pueda estudiar cómodamente sin distracciones.

- Establecer una rutina y usar una agenda para trabajar en las tareas y respetar los tiempos de descanso y alimentos.

- Explorar la plataforma en la cual se interactuará con el profesor y los compañeros.

- Informar al profesor si se tienen limitantes de acceso a internet.

- Revisar los criterios de evaluación y apoyarse en las rúbricas.

- Utilizar los recursos proporcionados por el profesor y buscar otras herramientas para complementar y fortalecer el aprendizaje.

- Tomar en cuenta la retroalimentación brindada por el profesor para enriquecer el aprendizaje.

- Dedicar tiempo para ejercitarse y emplear otras estrategias para despejar la mente cuando sea necesario.

Hay que recordar que la motivación, la autodisciplina y la honestidad son claves para el éxito del aprendizaje, por lo tanto, mantener una actitud positiva durante el curso en línea ayudará a lograr las metas, tanto en el plano académico como personal (Emagister, 2017; UANL, 2020).

\section{Incluir el factor de afectividad en un ambiente virtual}

La modalidad de cursos no presenciales requiere que se consideren, además de factores cognitivos, factores sociales y afectivos que permitirán que los estudiantes mantengan el interés y logren un aprendizaje significativo basado en la colaboración. Aunado a esto, sabemos que la preparación y actitud del profesor es fundamental para que un curso en línea funcione. Por tal motivo, la innovación 
y creatividad del docente juegan un papel esencial en el uso de herramientas y estrategias que generarán ambientes de aprendizaje propicios para el desarrollo de las competencias del curso (Quiñonez, Zapata y Canto, 2018).

El acompañamiento del docente es clave para fomentar el uso ético de las herramientas, el cumplimiento de las tareas y la generación de trabajo colaborativo. El profesor debe saber que su presencia en el curso en línea motivará a los estudiantes a tener comunicación constante a través de foros, chats, videollamadas y correos para hacerles sentir que no están solos y animarlos a expresar dudas, o bien situaciones que puedan poner en riesgo su desempeño académico. La responsabilidad del estudiante es un elemento inherente en el entorno virtual. Sin embargo, dada la situación que enfrentamos, la flexibilidad del docente en la entrega de tareas puede ayudar a disminuir ansiedad.

Estamos en tiempos en donde estaremos aprendiendo junto con los estudiantes a través de retos. Demostremos que nuestra prioridad son ellos y generemos grupos empáticos que logren en conjunto un aprendizaje significativo en un ambiente virtual.

\section{Involucrar a los estudiantes en su aprendizaje}

Una de nuestras primeras reacciones frente a la contingencia ha sido la de dar continuidad a nuestro curso. Lo hemos logrado mediante aplicaciones de interacción en tiempo real (síncronas) como Google Meet, Zoom, Webex, etcétera, con las que se reproduce, si bien de manera limitada, la forma en que se imparten las clases presenciales. El siguiente paso es dotar de interactividad a nuestros cursos con actividades que permitan a los estudiantes aplicar los contenidos de las clases y así asimilarlos de mejor forma. Para involucrar a los estudiantes en su aprendizaje, lo ideal es incorporar elementos de aprendizaje centrados en ellos, que les permitan realizar una actividad cognitiva con los conocimientos adquiridos, orientada a que apliquen lo que aprenden, socialicen entre sí sus impresiones y dudas, e incluso para que sean capaces de desarrollar sus capacidades analíticas o de ser creativos (Woods, 1994).

Otro elemento esencial es la retroalimentación, sea ésta de parte del profesor hacia ellos, entre los propios estudiantes, o, la que los estudiantes puedan ofrecerte también a ti sobre la forma en que el curso se desenvuelve, en este sentido es importante preguntarles cómo perciben la formación que reciben, con ello, además de darte elementos para mejorar o adecuar la clase logras que se vuelvan corresponsables con el desarrollo de la misma (ACUE, 2020; Flipped Learning Global Initiative, 2020).

Por último, dada la condición que se vive con la contingencia, la situación emocional de los estudiantes no es la mejor, el encierro y los cambios en su proceso educativo, así como la socialización con sus compañeros, se ha visto trastocada. Por 
ello, para garantizar su inclusión en el proceso educativo, es necesario mantener una comunicación activa y constante, con el fin de entender sus circunstancias y para que nadie deje de ser incluido en la clase (Meinecke, 2020).

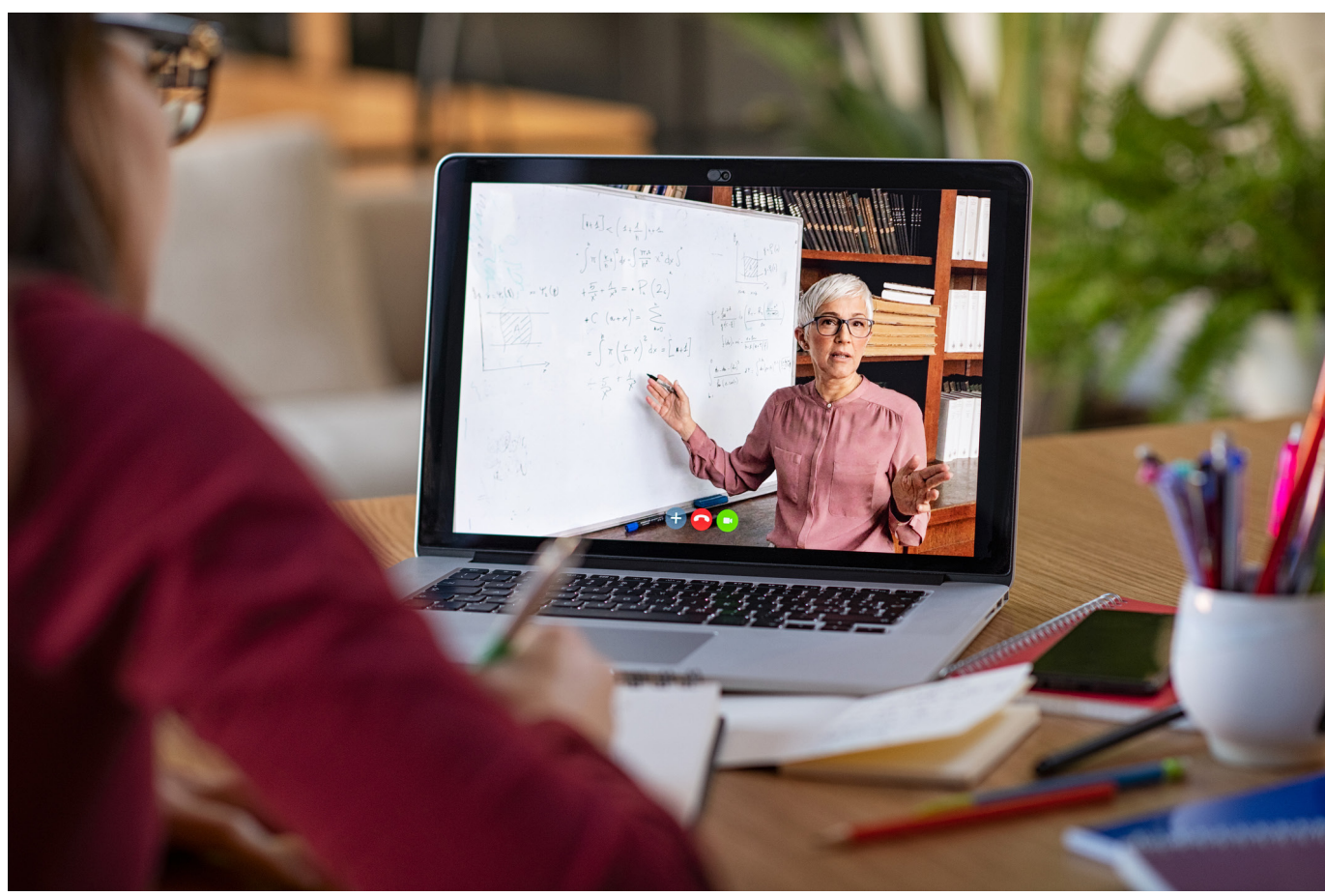

\section{Motivar a los estudiantes ante el cambio hacia la enseñanza a distancia}

Para motivar a los estudiantes a participar y estar atentos en las clases, es importante procurar mantener un estado de ánimo cordial y amigable en cada actividad que se realice con los estudiantes. Al hacerlo se ayuda a que los estudiantes se adapten mejor a esta modalidad, hay que recordar que estamos viviendo un momento extraordinario. También es relevante retomar análisis teóricos como los de Eggen y Kauckak (2009), que plantean la importancia de generar experiencias percibidas como desafiantes por el estudiante, que permitan que tenga cierto control y le generen curiosidad e interés. Esto seguramente repercutirá en la dinámica de la sesión y el logro de los objetivos. Para mantener motivados a los estudiantes sugerimos:

- Conocer muy bien la herramienta o medio que se utilizará, para apoyar la participación de los estudiantes. Esto ayudará a la fluidez de la clase y evitará distracciones por dificultades técnicas de uso de la herramienta.

- Diversificar los recursos digitales y las estrategias didácticas para evitar la monotonía. Presentar retos que motiven la participación activa de los estudiantes en el proceso de aprendizaje. 
- Respetar el horario estipulado y las fechas establecidas. Lo que permite apoyar en la organización del tiempo para actividades académicas y de descanso.

- Romper el hielo, mantener un ambiente tranquilo y empatizar con los estudiantes, tomando un momento para preguntar cómo están y escucharlos. Esto evitará que se sientan tensos durante la clase.

- Situarse en el mismo contexto cultural y social para captar la atención. Iniciar la clase con una actividad lúdica que se relacione con el tema, utilizando recursos que los estudiantes conozcan.

- Interactuar con los estudiantes durante la clase y fomentar su participación, ya que al no sentirse incluidos pueden distraerse fácilmente.

- Utilizar un lenguaje casual y respetuoso para que los estudiantes se sientan en una conversación de la que son parte importante.

- Asegurarse de realimentar las participaciones orales y escritas de los estudiantes, así como las entregas de tareas, ejercicios y exámenes. De esta manera, se mantendrá motvados a los estudiantes y se les acompañará en su proceso formativo, disminuyendo la sensación de abandono.

- Realizar un recuento de la clase con la participación de los estudiantes al terminar la sesión. Esta reseña puede ser de utilidad para iniciar la siguiente clase.

En esta transición un elemento clave será la motivación para aprender y, por supuesto, también la de enseñar. La motivación del profesor es indispensable y es necesario que tanto profesores como estudiantes mantengamos ese interés y gusto por enseñar y aprender aún en estas circunstancias.

\section{Establecer una relación profesor y estudiante efectiva en clases a distancia}

Es importante mantener una estrecha relación con los estudiantes para evitar que sientan que están solos con un dispositivo. Es sustancial que en este vínculo se perciba cercanía y apoyo (Quin, 2017), para ello sugerimos:

- Definir medios para comunicarse con ellos. Hacerles saber que pueden enviar comentarios o dudas de los temas o las tareas. Asimismo, permitir el envío de mensajes sobre sus experiencias y vivencias si así lo desean, para abrir un canal de cercanía personal. Sugerimos utilizar de preferencia medios grupales como redes sociales, pues el correo electrónico se podría saturar.

- Imprimir un toque de personalidad. Personalizar los materiales que se compartan. Por ejemplo, si se envía un archivo en formato PDF, realizar algunos comentarios sobre reacciones sorpresivas o interrogantes en algunos párrafos, con ello se dará la sensación de que se está con ellos. 
- Organizar el tiempo para responder los mensajes. Leérlos atentamente y enviar una respuesta alentadora. De acuerdo con la naturaleza del mensaje se puede enviar información de apoyo.

- Enviar mensajes alentadores. Hacerles saber a los estudiantes que se está siguiendo su progreso e invitarlos a hacer de la crisis una oportunidad para salir de la zona de comodidad en un continuo aprender y crecer, con ello se propiciará una sensación de seguridad óptima para el aprendizaje. Compartir pequeños desafíos y juegos mentales, notas sobre arte, cultura, música, comida, etcétera, que les permitan aprender de otros temas, y ser curiosos y creativos.

- Destinar tiempo para escuchar sus vivencias. Cuando se realicen las reuniones con los estudiantes de manera virtual se debe "romper el hielo" narrando alguna situación personal y lo que se está aprendiendo de esta situación, buscar que compartan alguna de sus experiencias y realizar comentarios alentadores y empáticos.

- Solicitar comentarios del curso. Esto permitirá detectar problemas y mejorar la dinámica y materiales sobre la marcha, también se les comunica a los estudiantes lo importante que es para ti lo que piensan y sienten. Se pueden utilizar diversos recursos para hacer encuestas.

\section{Referencias}

Association of College and University Educators (ACUE). (2020). ACUE'S online teaching toolkit. https://acue.org/online-teaching-toolkit/

* Caplan, G. (1961). An approach to community mental health. Grune \& Stratton.

* Eggen, P.y Kauckak, D. (2009). Estrategias docentes. Enseñanza de contenidos curriculares y desarrollo de habilidades de pensamiento ( $3^{a}$. ed.). Fondo de Cultura Económica.

* Emagister. (2017). TOP 10 consejos para realizar con éxito un curso virtual. https:// www.emagister.com/blog/10-consejos-realizar-exito-curso-virtual/

* Flipped Learning Global Initiative. (2020). RTOL: Rapid Transition to Online Learning. https://learn.flglobal.org/courses/rtol

- Meinecke, A. (2020). How to Make a Live Class Session More Effective and Run Smoother. https://observatory.tec.mx/edu-bits-2/make-a-live-class-sessionmore-effective-and-run-smoother

- Quin, D. (2017) Longitudinal and contextual associations between teacher-student relationships and student engagement: a systematic review. Review of educational Research, 87(2), 345-387. Dol: https://doi-org.pbidi.unam.mx:2443/10.3102/00346 54316669434 
* Quiñonez Pech, S. H., Zapata González, A., 7 Canto Herrera, P. J. (2018). Percepción de profesores sobre la afectividad en los entornos virtuales en una universidad pública del sureste de México. RIDE Revista Iberoamericana para la Investigación y el Desarrollo Educativo, 9(17), 195-224. Dol: https://doi.org/10.23913/ride.v9i17.378

* Universidad Autónoma de Nuevo León. (2020). Guía Práctica para Estudiantes. Dirección de Educación Digital. https://estrategia-digital.uanl.mx/estrategiadigital-estudiantes/

Woods. D.R. (1994). Problem-based Learning: How to Gain the Most from PBL. Griffing Printing Limited.

* Wosnitza, M. y Volet, S. (2005). Origin, direction and impact of emotions in social online learning. Learning and Instruction, 15(5), 449-464. Dol: https://doi. org/10.1016/j.learninstruc.2005.07.009

\section{Cómo CITAR ESTE ARTículo}

* Escamilla, J., García, R., Moreno, C., Osoria, J., Peña, A., González, A., Rodríguez, J., Salazar, L., Saldivar, R., Luna, V., Briz, O., García, A., Galván, N., Garrido, M., Infanzón, H., Rivero, C., Yap, K. y Zavala, C. (2020, septiembre-octubre). ¿Cómo abordar la dimensión socioafectiva en la enseñanza remota de emergencia?. Revista Digital Universitaria (RDU), 21(5). Dol: http://doi.org/10.22201/ cuaieed.16076079e.2020.21.5.10 